\title{
L'hérédo-dystrophie mouchetée du parenchyme cornéen
}

\author{
par \\ J. Francois et A. Neetens \\ (Gand)
}

Il y a quelques mois l'un de nous a décrit une nouvelle dystrophie hérédo-familiale de la cornée, l'hérédo-dystrophie nuageuse centrale du parenchyme cornéen. Il s'agit d'une dystrophie parenchymateuse, bilatérale et symétrique, dans laquelle le tiers central de la cornée est occupé par un disque de petits flocons nuageux grisâtres, sans structure apparente, à limites indécises. Ces flocons sont plus denses, plus volumineux et plus nombreux près de la membrane de Descemet, d'où ils diminuent progressivement aussi bien d'arrière en avant que du centre vers la périphérie. Tantôt ils occupent toutes les couches du stroma jusqu'à la membrane de Bowman; tantôt ils restent localisés aux couches profondes. A l'éclairage latéral on voit simplement que la partie centrale de la cornée est moins transparente et plus grise; il faut le biomicroscope pour reconnaître le détail des altérations. Celles-ci ne diminuent d'ailleurs pas la vision.

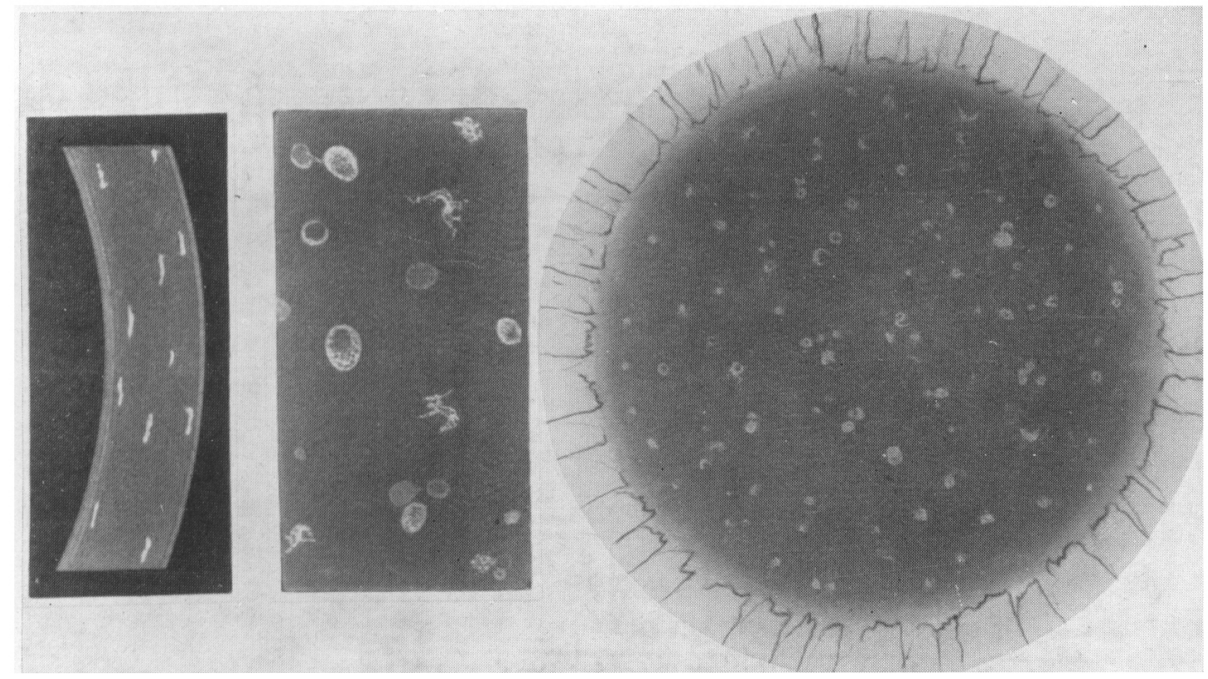

Fig. I 
Nous venons d'avoir l'occasion d'observer une autre variété d'hérédo-dystrophie parenchymateuse de la cornée, qui, elle non plus, n'a pas encore été décrite et que nous appellerons l'hérédo-dystrophie mouchetée du parenchyme cornéen (fig. I).

Cette dystrophie se caractérise par les signes suivants:

I. A l'examen ordinaire et à l'éclairage latéral on ne voit rien d'anormal. Ce n'est qu'au biomicroscope qu'on voit à l'intérieur du stroma cornéen se détacher très nettement de petites taches, plus ou moins opaques et plus ou moins nombreuses. Ces petites mouchetures ne se retrouvent pas seulement dans n'importe quelle couche du parenchyme, qu'elle soit postérieure, centrale ou antérieure, mais elles envahissent aussi toute l'étendue de la cornée et ne sont pas plus nombreuses au centre qu'à la périphérie. Entre les mouchetures le tissu cornéen est tout à fait intact et ne présente pas le moindre trouble.

2. Les mouchetures sont au moins partiellement opaques et, bien que minuscules, présentent des dimensions différentes de l'une à l'autre. Elle prennent des formes très variables, qui sont cependant du même type chez tous les sujets atteints. Les unes sont ovalaires, d'autres plus arrondies, d'autres encore très irrégulières ou festonnées. La densité de l'opacité est également variable: certaines taches sont plus opaques sur les bords et dessinent une bouée de sauvetage; d'autres présentent un trouble diffus et plus ou moins homogène; d'autres encore sont piquetées par des grains blancs, plus marqués à certains endroits qu'à d'autres. Leurs bords sont réguliers ou déchiquetés, mais toujours très nets. Examinées en coupe optique, on voit que toutes les taches sont aplaties et très peu épaisses.

3. Les altérations sont bilatérales et identiques des deux côtés.

4. Les membranes limitantes antérieures (épithélium et membrane de Bowman) ou postérieures (endothélium et membrane de Descemet) sont toujours absolument intactes. Il en est de même de la sensibilité cornéenne.

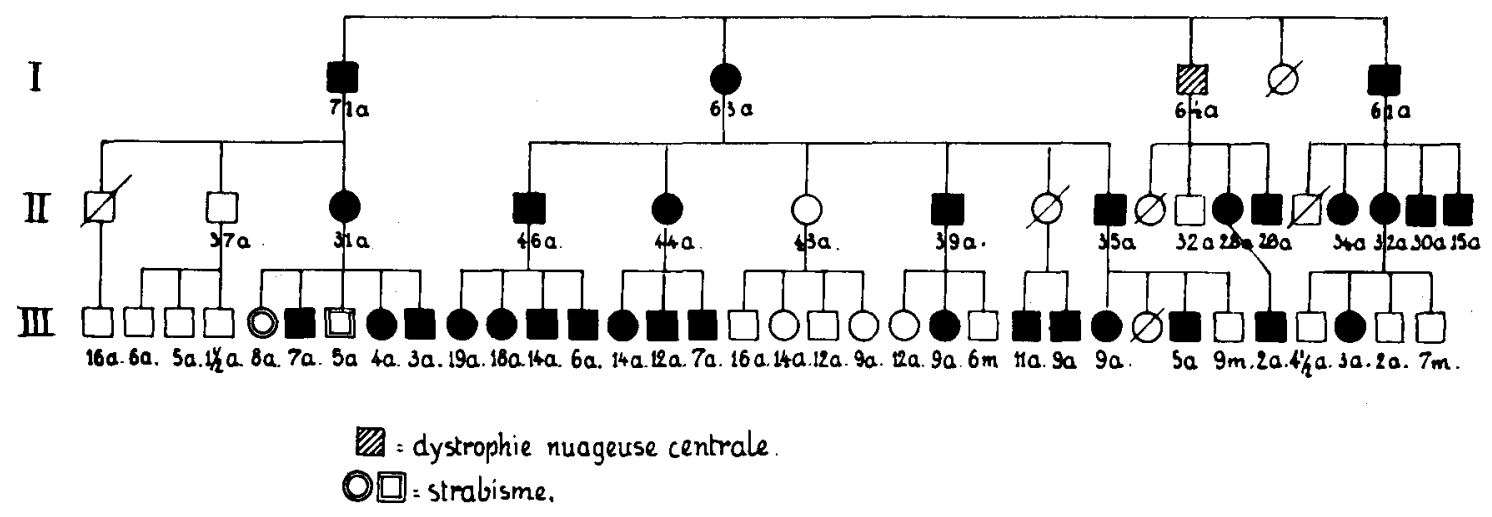

Iiig. 2 
5. La dystrophie mouchetée n'est en rapport avec aucune autre affection ou anomalie oculaire. Elle ne s'accompagne d'aucun phénomene inflammatoire. Elle ne diminue pas la vision, que nous avons toujours trouvée égale à I,o. Elle ne donne lieu à aucun trouble subjectif, à part peut-être parfois un certain degré de photophobic.

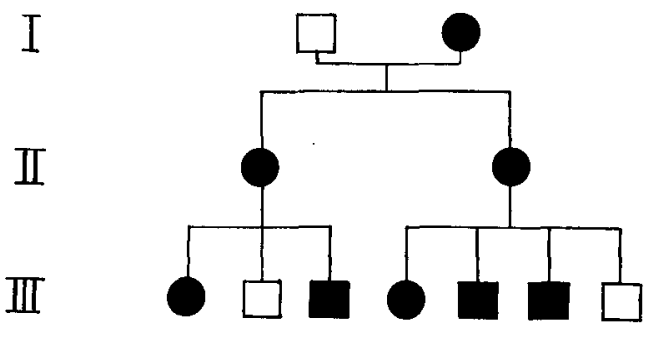

Fig. 3

6. Elle s'observe déjà chez les tout jeunes enfants, puisque nous l'avons rencontrée à deux et à trois ans; aussi pensons-nous qu'elle est congénitale ou en tout cas très précoce.

7. Elle n'est pas évolutive, puisque nous la retrouvons avec des caractères identiques chez les personnes âgées (63 et 7 I ans).

8. Elle n'est en corrélation avec aucune autre anomalie ou affection générale, tous les examens ayant été à ce point de vue négatifs.

9. Là dystrophie mouchetée du parenchyme cornéen se transmet de toute évidence suivant le mode dominant autosomique régulier (fig. 2). Nous l'avons en effet observée dans trois générations successives: 3 I sujets sur $5^{I}$ en sont atteints, soit $60 \%$. Il n'y a aucune prédilection pour l'un ou l'autre sexe, puisque nous trouvons I 3 femmes atteintes contre I 8 hommes atteints.

Tous les membres de la famille ont été examinés par nous-mêmes, à l'exception évidemment des six personnes décédées, sur lesquelles nous n'avons aucun renseignement.

La dystrophie mouchetée est identique chez tous les sujets atteints, mais le nombre des mouchetures est variable d'un sujet à l'autre: très nombreuses chez certains (I 7 sujets), elles le sont beaucoup moins chez d'autres (I4 sujets) et peuvent même se réduire à 4 ou 5 . Ce fait explique que la dystrophie paraît parfois plus prononcée et plus développée chez un enfant que chez un adulte.

Dans toute la famille nous n'avons trouvé aucune affection générale ni aucune autre anomalie oculaire, si nous exceptons deux cas de strabisme concomitant interne (III 5 et 7 ).

Un point intéressant, et sur lequel nous voulons attirer l'attention, est le fait que dans la première génération nous trouvons un homme ( I $_{3}$ ), qui présente une 
dystrophie nuageuse centrale du parenchyme cornéen. Cet homme a trois enfants vivants, dont deux sont atteints d'une dystrophie mouchetée, de sorte que nous devons nous demander s'il n'existe pas une parenté entre les deux variétés de dystrophie cornéenne et si la première ne peut pas constituer une variante de la deuxième (variabilité phénotypique du gène en cause?).

Nous avons eu l'occasion d'observer une deuxième famille, presentant la même dystrophie mouchetée du parenchyme cornéen (fig. 3): 8 personnes atteintes ( 5 femmes et 3 hommes) sur I I à travers 3 generations successives.

L'hérédo-dystrophie mouchetée du parenchyme cornéen ne peut être confondue avec aucune autre forme de dystrophie cornéenne. La seule, à laquelle on pourrait penser, est la dystrophie granuleuse (type I de Groenouw), qui se transmet également suivant le mode dominant et qui se caractérise par des opacités assez petites, blanchâtres, irrégulières, plutôt aplaties, nettement délimitées et se détachant dans un stroma cornéen par ailleurs bien transparent.

Il y a cependant des différences nombreuses et essentielles:

I. La dystrophie granuleuse débute plus tardivement et au plus tôt généralement vers l'âge de 5 ans.

2. Les couches profondes et la périphérie de la cornée restent intactes.

3. Les altérations sont visibles à l'œil nu.

4. Elles sont progressives et altèrent la vision parfois assez rapidement (à partir de l'âge de 20 ans).

5. La surface cornéenne perd son aspect lisse et luisant.

6. La sensibilité de la cornée est touchée. 


\section{Résumé}

Les auteurs décrivent une nouvelle dystrophie hérédo-familiale de la cornée, l'hérédo-dystrophie mouchetée du parenchyme cornéen. A l'intérieur du stroma cornéen des deux yeux se détachent très nettement de petites mouchetures plus ou moins nombreuses, qui se retrouvent dans toutes les couches du parenchyme et aussi bien au centre qu'à la périphérie. La dimension, la forme et la densité des taches sont très variables: elles sont ovulaires, arrondies ou festonnées; certaines sont plus opaques sur les bords; d'autres présentent un trouble plus diffus; d'autres encore sont piquetées; leurs bords sont nets, réguliers ou déchiquetés. En coupe optique les opacités sont minces et aplaties. Cette dystrophie n'est pas évolutive et se transmet suivant le mode dominant autosomique régulier. Un membre d'une des familles décrites présente une dystrophie nuageuse centrale du parenchyme cornéen.

\section{Bibliographie}

FrançoIs, J.: Dystrophies hérédo-familiales de la cornée. Journ. Génét. hum., I, Io3, I952.

_- Une nouvelle dystrophie hérédo-familiale de la cornée. Journ. Génét. hum., 5, I89, I956.

\section{RIASSUNTO}

Gli AA. descrivono una nuova distrofia erodo-familiare della cornea, l'eredo-distrofia punteggiata del parenchima corneale. Nell'interno dello stroma corneale dei due occhi si evidenziano molto nettamente delle piccole punteggiature più o meno nume- rose che si riscontrano in tutti gli strati del parenchima, tanto al centro come alla periferia. La dimensione, la forma e la densità delle macchie sono molto variabili: esse possono essere ovulari, arrotondate $o$ festonate; alcune sono più opache sui bordi, altre presentano un'opacità diffusa; altre ancora sono punteggiate; $i$ lo- ro bordi sono netti, regolari o sfrangiati. In sezione ottica le opacità sono sottili e appiattite. Quest I distrofia non è evolutiva e si trasmette seguendo il modulo dominante autosomico regolare. Un membro di una delle famiglie descritte presenta una distrofia nebulosa centrale del parenchima corneale. 


\section{SUMMARY}

The authors describe a new hereditary dystrophy of the cornea, the specked heredo-dystrophy of the corneal parenchyma. In the corneal stroma of both eyes speckles can be easily distinguished; they are more or less numerous and can be found in all the layers of the parenchyma,

\section{ZUSAMMENFASSUNG}

Die Autoren beschreiben eine neue heredo-familiäre Dystrophia der Hornhaut: die gesprenkelte heredo-Dystrophia des Hornhautparenchyms. Im inneren des Hornhaut-stromas beider Augen zeichnen sich sehr deutlich k!eine mehr oder weniger zahlreiche Flecke; diese befin- as well in the center as at the periphery. Dimension, shape and density of the speckles are very variable: they are oval, round or festooned; some of them are opaque at the borders; others show a more diffuse trouble; others are dotted; their borders are well-cut, regular or indented. In optical section the

den sich in allen Schichten des Parenchyms und ebenso wohl in dem Mitten wie an der Peripherie. Die Ausdehnung, die Form und die Dichtigkeit der Flecke sind sehr verschieden: sic sind oval, rund oder festoniert. Gevisse sind mehr undurchsichtig am Rände; andere sind mehr allgemein getrübt; sichere sind punktiert. Ihre Rände sind opacities are thin and flat. This dystrophy is not evolutive and is transmitted as an autosomal regular dominant trait. One member of the affected family shows a cloudy central dystrophy of the corneal stroma, so that it is possible that there exists a relationship between these two affections.

deutich, regelmässig oder zerstückelt. Im optischen Schnitt sind die Flecke dünn und $a b-$ geplattet. Die Dystrophie ist nicht fortschreitend und wird übertragen nach dem regelmässig autosomalen dominanten Modus. Ein Mitglied der beschriebenen Familie zeigt eine zentrale wolkige Dystrophie des Hornhautparenchyms. 